\title{
Anatomo-Clinical Case: Coexistence of Tuberculosis with Axillary Lymph Node Metastasis in Breast Carcinoma
}

\author{
Aimé Sosthène Ouédraogo ${ }^{1,2 *}$, Hierrhum Aboubacar Bambara ${ }^{3}$, \\ Franck Auguste Hermann Ademayali Ido1, Welbnoaga Norbert Ramdé2,4, \\ Rimwaogdo Jeremie Sawadogo ${ }^{5}$, Ibrahim Savadogo6, Souleymane Ouattara7, \\ Hassami Barry1, Assita Sanou-Lamien ${ }^{2,8}$, Olga Melanie Lompo ${ }^{2,8}$ \\ ${ }^{1}$ Pathological Anatomy Unit of Bogodogo District Hospital, Ouagadougou, Burkina Faso \\ ${ }^{2}$ University Department of Research and Training in Health Sciences (UFR/SDS), Ouagadougou, Burkina Faso \\ ${ }^{3}$ Clinical Oncology and Hematology Unit of Bogodogo District Hospital, Ouagadougou, Burkina Faso \\ ${ }^{4}$ Legal Medicine Unit of Bogodogo District Hospital, Ouagadougou, Burkina Faso \\ ${ }^{5} \mathrm{MD}, \mathrm{MPH}, \mathrm{CPH}$ Research Institute, JPS Health Network, Fort Worth, TX, USA \\ ${ }^{6}$ Pathological Anatomy Unit of Ouahigouya Regional Hospital Centre, Ouagadougou, Burkina Faso \\ ${ }^{7}$ Pathological Anatomy Unit of Blaise Compaoré University Hospital Centre, Ouagadougou, Burkina Faso \\ ${ }^{8}$ Yalgado Ouedraogo University Hospital Centre, Ouagadougou, Burkina Faso \\ Email: *sostheneaime@yahoo.fr
}

How to cite this paper: Ouédraogo, A.S., Bambara, H.A., Ido, F.A.H.A., Ramdé, W.N., Sawadogo, R.J., Savadogo, I., Ouattara, S., Barry, H., Sanou-Lamien, A. and Lompo, O.M. (2018) Anatomo-Clinical Case: Coexistence of Tuberculosis with Axillary Lymph Node Metastasis in Breast Carcinoma. Open Journal of Pathology, 8, $132-138$.

https://doi.org/10.4236/ojpathology.2018.8 $\underline{4015}$

Received: August 2, 2018

Accepted: September 22, 2018

Published: September 25, 2018

Copyright $\odot 2018$ by authors and Scientific Research Publishing Inc. This work is licensed under the Creative Commons Attribution International License (CC BY 4.0).

http://creativecommons.org/licenses/by/4.0/

cC) (i) Open Access

\begin{abstract}
Introduction: The coexistence of tuberculosis with axillary lymph node metastasis in breast carcinoma is uncommon. Observation: We report a case of a patient aged 59 years presenting a painless nodule in the right breast for one year. The scan and mammography revealed a long-axis node of $3 \times 2 \times 1 \mathrm{~cm}$ in the upper outer quadrant of the right breast ranked stage IV by the American College of Radiology (ACR), associated with a set of axillary lymph nodes and the largest one measuring $15 \times 15 \times 20$ millimeters $(\mathrm{mm})$. The breast biopsy helped diagnose a Scarff Bloom Richardson (SBR) grade II non-specific invasive carcinoma, modified by Ellis and Elston. A right mastectomy associated with a lymph node dissection was performed. We noticed a not well defined and whitish $5 \mathrm{~mm}$ tumor mass associated with 16 lymph nodes removed. The histological examination confirmed the diagnosis of SBR grade II non-specific invasive carcinoma with invasion of 7 lymph nodes $(\mathrm{N}+=7 / 16)$. In 3 metastatic lymph nodes, there were epithelioid and gigantocellular granulomas with full central necrosis. The Ziehl Neelsen staining had highlighted acid-fast bacilli. The tumor was oestrogen and progesteron receptor, without an overexpression of the oncoprotein human epidermal growth factor receptor 2 (HER2), which corresponds to a 0 score and the Ki 67 prolife-
\end{abstract}


ration index assessed at $10 \%$. The patient was given an anti-tuberculosis treatment combining Rifampicin (H), Isoniazid (I), Pyrazinamid (Z), Ethambutol (E) over 2 months and secondly a combination of Rifampicin and Isoniazid over 4 months (2RHZE/4 RH). The anti-tumor chemotherapy used a protocol combining 3 FAC60+ 3 Docetaxel $\left(\mathrm{F}=\right.$ Fluorouracil $^{\circledR} ; \mathrm{A}=$ Adriblastin $^{\circledR}, \mathrm{C}=$ Cyclophosphamid). Conclusion: This coexistence is uncommon, of incidental discovery and necessitates a multidisciplinary care.

\section{Keywords}

Breast Carcinoma, Tuberculosis, Lymph Node, Histopathology

\section{Introduction}

The coexistence of breast cancer and tuberculosis is very uncommon and less reported in the literature [1] [2] [3] [4] [5]. The clinical features of mammary tuberculosis may reveal nodules and ulcerations with the appearance of lymphadenopathies leading us to suspect a malign tumor. It is the histological examination that enabled making the differential diagnosis. We report an uncommon case of non-specific infiltrating breast carcinoma with metastatic homolateral lymph node associated with lymph node tuberculosis that was incidentally discovered during the histological examination.

\section{Anatomo-Clinical Case}

We report the case of a patient of 59 years, 7 pregnancies, 5 parities, menopausal since 12 years, without cancer disease history in her family, who presented a painless and moving nodule discovered in the right breast after a self-breast examination. The nodule had been evolving for one year. The scan and mammography revealed a nodule of $3 \times 2 \times 1 \mathrm{~cm}$ in the upper outer quadrant of the right breast ranked ACR IV, associated with a group of axillary lymph nodes including the largest one measuring $15 \times 15 \times 20 \mathrm{~mm}$. A breast biopsy had helped diagnose a Scarff Bloom Richardson (SBR) grade II non-specific invasive breast carcinoma, modified by Ellis and Elston.

A right mastectomy associated with a lymph node dissection was performed and presented a not well defined and whitish $5 \mathrm{~mm}$ tumor mass of large axis with 16 lymph nodes.; the largest one was measuring $3 \mathrm{~cm}$.

The histological examination had confirmed the diagnosis of SBR grade II non-specific invasive breast carcinoma with an invasion of 7 lymph nodes over $16(\mathrm{~N}+=7 / 16)$. In 3 metastatic lymph nodes, there were epithelioid and gigantocellular granulomas centered by a full necrosis (Figure 1 and Figure 2). The Ziehl Neelsen staining had highlighted acid-fast bacilli (AFB) (Figure 3). There were no vascular emboli or peripheral nerve sheathing.

The immunohistochemistry examination carried out in paraffin section had revealed a high expression of estrogen and progesterone receptors assessed at 


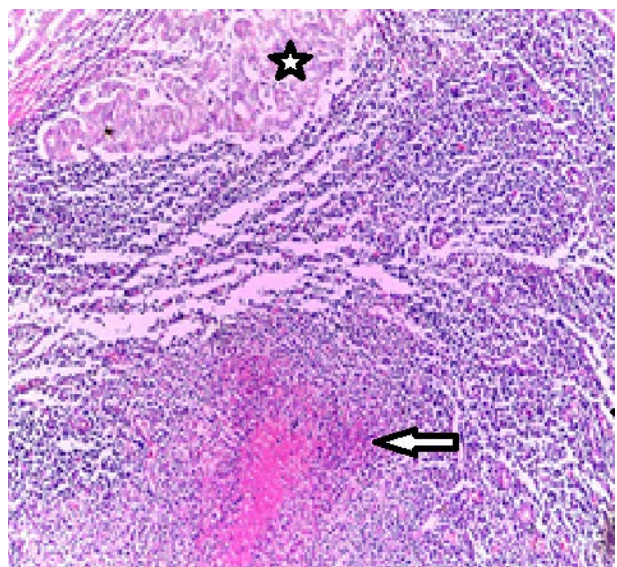

Figure 1. Breast carcinoma and lymph node tuberculosis. Hematein Eosin (HE) stain, Magnification $\times 100$. Carcinomatous proliferation (star) infiltrating a lymph node parenchyma which is the site of a granulomatous inflammatory reaction with central necrosis (arrow).

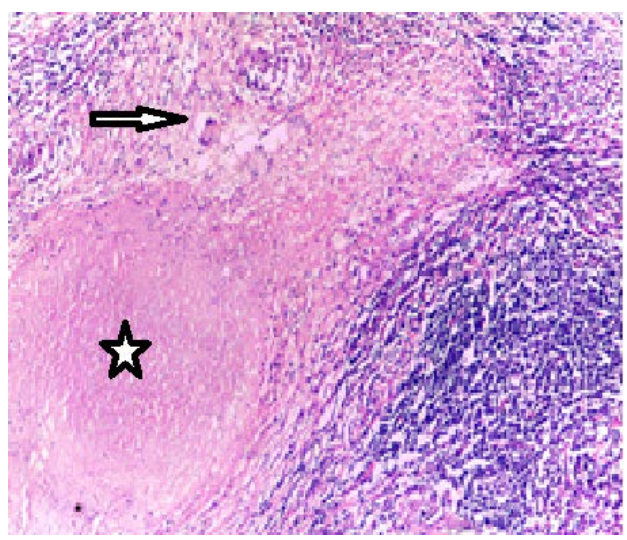

Figure 2. Breast carcinoma and lymph node tuberculosis. Hematein Eosin (HE) stain, Magnification $\times 100$. Granulomatous inflammatory reaction with complete central necrosis (star) and giant Langhans cells (arrow).

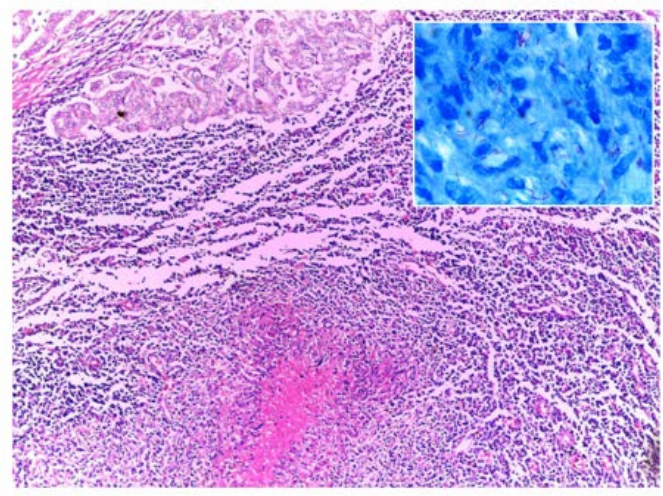

Figure 3. Breast carcinoma and lymph node tuberculosis. Hematein Eosin (HE) stain, Magnification $\times 100$ and in cartridge Zielh Neelsen staining. Carcinomatous proliferation infiltrating a lymph node parenchyma which is the site of a granulomatous inflammatory reaction with central necrosis and highlight of Acid-Fast Bacilli (AFB) with Zielh Neelsen staining (cartridge). 
90\% each (Figure 4 and Figure 5), without an overexpression of the oncoprotein HER2 (Score 0). The Ki 67 proliferation index was assessed at 10\% (Figure 6).

The patient was submitted to an anti-tuberculosis treatment associated with a chemotherapy protocole made up of 3 FAC $60+3$ Docetaxel. The FAC60 protocol followed by the Docetaxel protocol was given on a regular interval of 21 days at the same time with the anti-tuberculosis protocol: $2 \mathrm{RHZE} / 4 \mathrm{RH}(\mathrm{R}=\mathrm{Rifam}$ picin; $\mathrm{H}$ = Isoniazid; $\mathrm{Z}=$ Pyrazinamid; $\mathrm{E}=$ Ethambutol). The major side

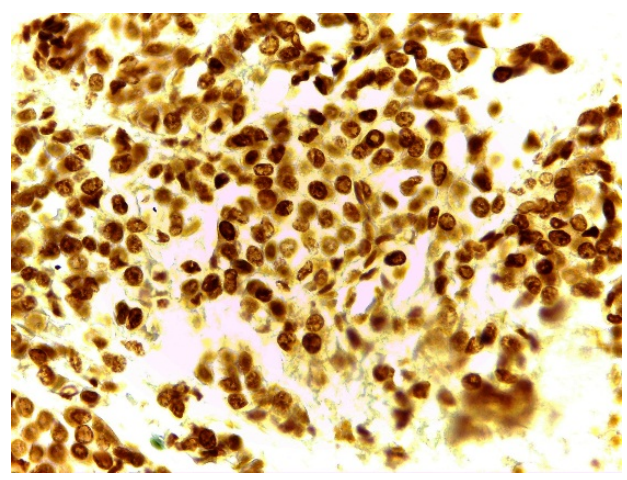

Figure 4. Breast carcinoma and lymph node tuberculosis. Immunohistochemistry Magnification $\times 400$. Intense and diffuse immunostaining with anti-estrogen receptor $(\mathrm{RO})$ antibodies relevant to tumor cell nuclei.

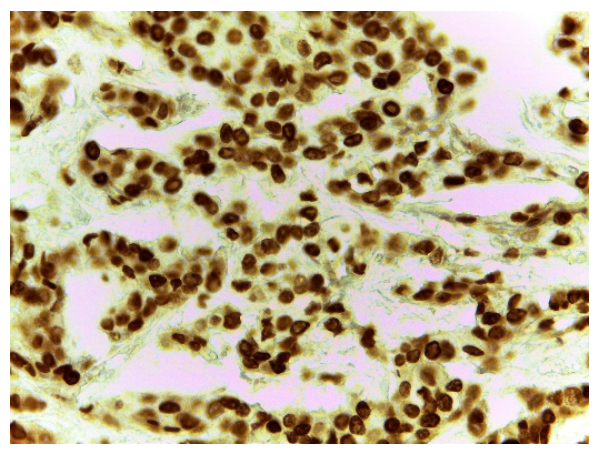

Figure 5. Breast carcinoma and lymph node tuberculosis. Immunohistochemistry Magnification $\times 400$. Intense and diffuse immunostaining with anti-progesterone receptor (RP) antibodies relevant to tumor cell nuclei.

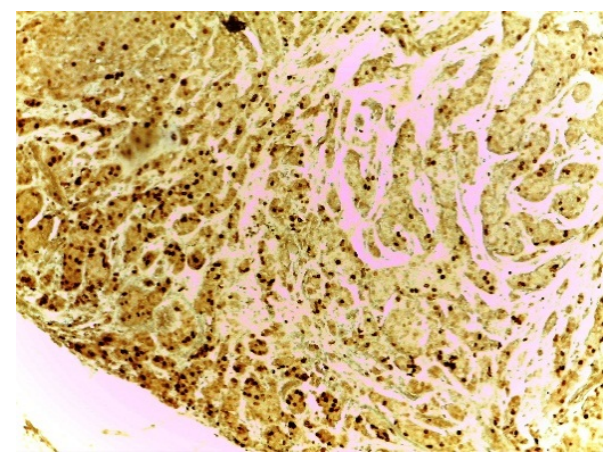

Figure 6. Breast carcinoma and lymph node tuberculosis. Immunohistochemistry Magnification $\times 400$ (H \& E). Moderate and diffuse immunostaining with anti-Ki 67 antibody showing a proliferation index evaluated at $10 \%$. 
effect was a clinical anemia with a hemoglobin rate at $10.5 \mathrm{~g} / \mathrm{ml}$. This anemia was contained through the administering of anti-anemia drugs. The patient is at her $5^{\text {th }}$ chemotherapy cycle.

\section{Discussion}

The coexistence of breast cancer and tuberculosis is very uncommon and less reported in the literature. This coexistence raises difficulties in the diagnostic and therapeutic care [3]. The first case was described in 1899 by Wharti, later followed by other authors [6]. The prevalence is estimated at 19/10,000 according to the data in the literature [7]. Extra-pulmonary tuberculosis most often localizes in cervical, inguinal and axillary lymph nodes, but the coexistence with a cancer is very rare and of incidental discovery [6]. The histological examination of the removed lymph nodes revealed a non-specific invasive breast carcinoma associated with an epithelioid and gigantocellular granuloma with full central necrosis which is characteristic of tuberculosis. The giant cells are of Langhans type (Figure 2). The Ziehl Nielsen staining often highlighted AFBs (Figure 3). The histology enabled determining the conventional prognosis factors, especially the Nottingham score (SBR grade II), the number of lymph nodes invaded over the total number of lymph nodes removed $(\mathrm{N}+=7 / 16)$ and the presence of vascular emboli. Other prognostic and therapeutic factors are determined by the immunohistochemistry with the dosage of estrogen (RO) and progesterone (RP) receptors, the HER2 oncoprotein and the Ki67 proliferation index. These elements have helped establish a molecular classification of the tumor as Luminal A (RO+, RP+, HER2-, Ki67 inferior to 14\% [8].

The patient was submitted to an anti-tuberculosis treatment associated with a chemotherapy protocol. The anti-tuberculosis protocol is based on polychemotherapy. The first-line drugs of tuberculosis used by the National Tuberculosis Plan (PNT) of Burkina Faso are five in number: Isoniazid H, Rifampicin R, Streptomycin S, Ethambutol E, Pyrazinamid Z.

The treatment is made up of two phases. An initial phase of treatment or intensive phase comprises four drugs that are Ethambutol (E), Rifampicin (R), Isoniazid $(\mathrm{H})$ and Pyrazinamid $(\mathrm{Z})$. The advantage of this phase is that it is very efficient in reducing bacilli and the resistance to anti-tuberculosis drugs. It takes two or three months. The continuation phase is necessary for the patient's final recovery. It helps avoid the recurrence of tuberculosis when the treatment is stopped. It required 2 drugs over a period of four months.

Tolerance and compliance with the treatment were good. They were regularly assessed through the Directly Observed Therapy (DOT) strategy. This strategy is recommended by WHO and consists in giving the drugs under control or verifying at least 3 times a week if they are effectively taken.

Chemotherapy is used as adjuvant and involves the $3 \mathrm{FAC}+3$ Docetaxel protocol. It is currently recommended to give it before the radiotherapy. The treatment usually takes six months. 
Our patient did not benefit from radiotherapy and hormonotherapy, due to the lack of financial means. The 2 protocols (anti-tuberculosis treatment and chemotherapy) were given at the same time. There is no consensus over the simultaneous or separated combination of these two protocols. Anti-tuberculosis treatment is a determining factor in cancer prognosis and evolution. Thus, anti-bacilli treatment must be initiated once the diagnosis is made, as there is a risk for the occurrence of serious forms of tuberculosis (miliary and meningitis tuberculosis) as the consequence of the immunodepression caused by the anti-cancer treatment [9]. However, the combination of the anti-tuberculosis treatment with anti-cancer chemotherapies may entail a multiplication of the risk of adverse effects through an exacerbation of toxicities or a modification in the efficiency of the anti-cancer treatment 10]. The side effect noted was a clinical anemia with a hemoglobin rate at $10.5 \mathrm{~g} / \mathrm{dl}$, addressed through an anti-anemia treatment.

The evolution still remains favourable without recurrence of cancer or the appearance of a tuberculosis disease.

\section{Conclusion}

This coexistence is uncommon and of incidental discovery, hence there is a need for a careful and full examination of lymph node dissections. The treatment requires a multidisciplinary collaboration.

\section{Conflicts of Interest}

The authors declare no conflict of interest.

\section{References}

[1] Pujani, M., Khan, S., Hassan, M.J., Jetley, S. and Raina, P.K. (2015) Coexistence of Metastatic Breast Carcinoma and Primary Tuberculosis in Axillary Lymph Nodes: A Report of a Rare Case. Breast Disease, 35, 195-198. https://doi.org/10.3233/BD-150405

[2] Bromberg, S.E. and Tenorio do Amaral, P.G. (2015) Tuberculosis Axillary Lymph Node Coexistent Breast Cancer in Adjuvant Treatment: Case Report. Einstein, 13, 423-425. https://doi.org/10.1590/S1679-45082015RC2963

[3] Pandey, P., Dixit, A., Tanwar, A. and Mahajan, N.C. (2014) Infiltrating Ductal Carcinoma Breast, Metastatic to Ipsilateral Axillary Lymph Nodes Harboring Primary Tuberculosis Lymphadenitis: A Case Report. Pan African Medical Journal, 18, 167. https://doi.org/10.11604/pamj.2014.18.167.1494

[4] Bansal, A., Sipayya, V., Chintamani, P. and Saxena, S. (2015) Carcinoma and Tuberculosis on the Breast Coexisting in Same Breast with Axillary Lymphadenopathy: A Rare Association. Indian Journal of Cancer, 52, 229-230. https://doi.org/10.4103/0019-509X.175818

[5] Baslaim, M.M., Al-Amoudi, A.S., Al-Ghamdi, A.M., Ashour, A. and Al-Numani, S.T. (2013) Case Report: Breast Cancer Associated with Contralateral Tuberculosis of Axillary Lymph Nodes. Word Journal of Surgical Oncology, 11, 43. https://doi.org/10.1186/1477-7819-11-43

[6] Avninder, S. and Saxena, S. (2006) Infiltrating Ductal Carcinoma of the Breast, Me- 
tastatic to Axillary Lymph Node to Harboring Primary Tuberculosis Lymphadenitis. Pathology Oncology Research, 12, 188-189. https://doi.org/10.1007/BF02893368

[7] Pandey, M., Abraham, K.E., Chandramohan, K. and Rajan, B. (2003) Tuberculosis and Metastatic Carcinoma Coexistence in Axillary Lymph Node: A Case Report. Word Journal of Surgical Oncology, 1, 1-3. https://doi.org/10.1186/1477-7819-1-1

[8] Camillo, A. and Thomas, P. (2014) Mémento de Pathologie. 4th Edition, Vernazobres-Grego, Paris, 574.

[9] Frappaz, D., Biron, P., Biron, E., Amrane, A., Philip, T. and Brunat-Mentigny, M. (1984) Severe Neurological Toxicity Secondary to the Association of Accidental Poisoning by Isionazid and a Protocol Containing High Dose of Vincristine. Pediatrie, 39, 133-140. 